\title{
On the Stability of Compositions of Universally Stable, Greedy Contention-Resolution Protocols
}

\author{
D. Koukopoulos ${ }^{1}$, M. Mavronicolas ${ }^{2}$, S. Nikoletseas ${ }^{1}$, and P. Spirakis ${ }^{1 \star}$
}

1 Department of Computer Engineering \& Informatics, University of Patras and Computer Technology Institute (CTI), Riga Fereou 61, P. O. Box 1122, 26110

Patras, Greece. \{nikole,koukopou, spirakis\}@cti.gr

2 Department of Computer Science, University of Cyprus, 1678 Nicosia, Cyprus.

mavronic@ucy.ac.cy

\begin{abstract}
A distinguishing feature of today's large-scale platforms for distributed computation and communication, such as the Internet, is their heterogeneity, predominantly manifested by the fact that a wide variety of communication protocols are simultaneously running over different distributed hosts. A fundamental question that naturally poses itself concerns the preservation (or loss) of important correctness and performance properties of the individual protocols when they are composed in a large network. In this work, we specifically address stability properties of greedy, contention-resolution protocols operating over a packet-switched communication network.

We focus on a basic adversarial model for packet arrival and path determination for which the time-averaged arrival rate of packets requiring a single edge is no more than 1. Stability requires that the number of packets in the system remains bounded, as the system runs for an arbitrarily long period of time. It is known that several commonly used contentionresolution protocols, such as LIS (Longest-in-System), SIS (Shortest-inSystem), NTS (Nearest-to-Source), and FTG (Furthest-to-Go) are universally stable in this setting - that is, they are stable over all networks. We investigate the preservation of universal stability under compositions for these four greedy, contention-resolution protocols. We discover:

- The composition of any two protocols among SIS, NTS and FTG is universally stable.

- The composition of LIS with any of SIS, NTS and FTG is not universally stable: we provide interesting combinatorial constructions of networks over which the composition is unstable when the adversary's injection rate is at least 0.519 .

- Through an involved combinatorial construction, we significantly improve the current state-of-the-art record for the adversary's injection rate that implies instability for FIFO protocol to 0.749 . Since 0.519 is significantly below 0.749 , this last result suggests that the potential for instability incurred by the composition of two universally stable protocols may be worse than that of some single protocol that is not universally stable.
\end{abstract}

* This work has been partially supported by the IST Program of the European Union under contract numbers IST-1999-14186 (ALCOM-FT) and IST-2001-33116 (FLAGS). 


\section{Introduction}

\subsection{Motivation-Framework}

Heterogeneous Networks. A key feature of contemporary large-scale platforms for distributed communication and computation, such as the Internet, is their heterogeneity. Heterogeneity comes around in many different flavors. For example, the specifics of how the computers in different parts of the network are connected (directly or indirectly) with each other, and the properties of the links that foster the interconnection, is difficult to characterize uniformly. Second, different traffic sources over the Internet (due to varying mechanisms for supporting different classes and qualities of service) result in a heterogeneous mix of traffic traces. Third but not least, although, conceptually, the Internet uses a unified set of protocols, in practice each protocol has been implemented with widely varying features (and of course bugs). (See the recent interesting article by Floyd and Paxson [7] for an extended discussion on the heterogeneity of Internet.) Thus, heterogeneity is a crucial feature that makes it difficult to model, verify and analyze the behavior of such large-scale communication networks.

Objectives. In this work, we embark on a study of the impact of heterogeneity of distributed systems on their correctness and performance properties. More specifically, we wish to pose the general question of which correctness and performance properties of individual, different modules of a distributed system are maintained and which are not when such modules are composed into a larger, heterogeneous distributed system. We choose, as a test-bed, the case of distinct communication protocols that are simultaneously running on different hosts in a distributed system. We ask, in particular, which (and how) stability properties of greedy, contention-resolution protocols operating over a packet-switched communication network are maintained under composition of such protocols.

Framework of Adversarial Queueing Theory. We consider a packetswitched communication network in which packets arrive dynamically at the nodes with predetermined paths, and they are routed at discrete time steps across the edges. We focus on a basic adversarial model for packet arrival and path determination that has been recently introduced in a pioneering work by Borodin et al. 3], under the name Adversarial Queueing Theory. Roughly speaking, this model views the time evolution of a packet-switched communication network as a game between an adversary and a protocol. At each time step, the adversary may inject a set of packets into some nodes. For each packet, the adversary specifies a simple path (including an origin and destination) that the packet must traverse; when the packet arrives to destination, it is absorbed by the system. When more than one packets wish to cross a queue at a given time step, a contention-resolution protocol is employed to resolve the conflict. A crucial parameter of the adversary is its injection rate $r$, where $0<r<1$. Among the packets that the adversary injects in any time interval $I$, at most $\lceil r|I|\rceil$ can have paths that contain any particular edge. Such a model allows for adversarial injection of packets, rather than for injection according to a randomized, oblivious process (cf. [4]). 
Table 1. Contention-resolution protocols considered in this paper. (US stands for universally stable)

\begin{tabular}{||l|l|l||}
\hline Protocol name & Which packet it advances: & US \\
\hline \hline Shortest-in-System (SIS) & The most recently injected packet into the network & $\sqrt{ }$ \\
\hline Longest-in-System (LIS) & The least recently injected packet into the network & $\sqrt{ }$ \\
\hline Furthest-to-Go (FTG) & The furthest packet from its destination & $\sqrt{ }$ \\
\hline Nearest-to-Source (NTS) & The nearest packet to its origin & $\sqrt{ }$ \\
\hline First-In-First-Out (FIFO) & The earliest arrived packet at the queue & X \\
\hline \hline
\end{tabular}

Stability. Stability requires that the number of packets in the system remains bounded, as the system runs for an arbitrarily long period of time. Naturally, achieving stability in a packet-switched communication network depends on the rate at which packets are injected into the system, and on the employed contention-resolution protocol. Till our work, the study of stability has focused on homogeneous networks, that is, on networks in which the same contentionresolution protocol is running at all queues. In this work, we embark on a study of the effect of composing contention-resolution protocols on the stability of the resulting system. (By composition of contention-resolution protocols, we mean the simultaneous use of different such protocols at different queues of the system.)

Greedy Contention-Resolution Protocols. We consider only greedy protocols- ones that always advance a packet across a queue (but one packet at each discrete time step) whenever there resides at least one packet in the queue. The protocol specifies which packet will be chosen. We study five greedy protocols (all of which enjoy simple implementations): Say that a protocol is stable [3] on a given network if it induces a bounded number of packets in the network against any adversary with injection rate less than 1 . (Note that the bound may depend on parameters of the network.) The first four of these protocols (namely, SIS, LIS, FTG and NTS) are universally stable- each is stable on all networks [1. Section 2.1]. In contrast, FIFO (one of the most popular queueing disciplines, because of its simplicity) is not universally stable [1 Theorem 2.10]. Approach. We consider all combinations of two from the four universally stable protocols, and we examine whether the corresponding composition is universally stable. We either show that it is, or we demonstrate a network and an adversary (with some specific injection rate less than 1) such that the composition is not stable on the network (against the adversary). In addition, in order to qualitatively evaluate how unstable are the compositions that turn out not to be universally stable, we also consider the FIFO protocol, which is known not be universally stable; we measure the instability of the composition against that of FIFO by establishing the best lower bound we can on the adversary's injection rate that implies instability for the composition and for FIFO, and we compare the two resulting lower bounds. 


\subsection{Contribution}

Summary of Results. In this work, we initiate the study of the stability properties of heterogeneous networks with compositions of greedy contention-resolution protocols, such as SIS, LIS, FTG, NTS and FIFO, running on top of them. Our results are three-fold; they are summarized as follows:

- We establish universal stability for compositions of any two among the (universally stable) SIS, FTG, and NTS protocols (Theorem 1).

- We establish that, surprisingly, the composition of LIS with any of SIS, NTS and FTG is not universally stable (Theorem 2).

To show this, we provide interesting combinatorial constructions of networks, for each queue of which we specify the contention-resolution protocol to be used, so that the composition of the protocols is unstable if the injection rate of the adversary is at least 0.519 .

- We establish a new lower bound on the instability threshold of the FIFO protocol. More specifically, we provide an involved combinatorial construction of a network containing only FIFO queues and an adversary with injection rate 0.749 that result to instability (Theorem 3 ).

This result not only significantly improves the current record (that is, the lowest known) instability threshold for FIFO [5. Theorem 3.1]. More importantly perhaps, it provides, as we argue, a standard for evaluating the lower bound (0.519) on the instability thresholds we established for the not universally stable compositions (Theorem 21). Since 0.519 is substantially less than 0.749 (in the climax [0.1]), and since lowering the instability threshold for FIFO has undergone a series of subsequent improvements in recent papers in the literature [1815] culminating to the 0.749 shown in this work, these together may modestly suggest that composing two universally stable protocols may, surprisingly, turn out to exhibit more unstable behavior than a single protocol that is already known to not be universally stable (such as FIFO).

The combinatorial constructions of networks and adversaries that we have employed for showing that certain compositions of universally stable protocols are not universally stable significantly extend ones that appeared before in 113 , 5. In more detail, some of the tools we devise in order to obtain constructions of networks and adversaries that imply improved bounds are the following:

- We employ combinatorial constructions of networks with multiple "parallel" paths between a common origin and destination; we judiciously use such paths for the simultaneous injection of various non-overlapping flows.

- We introduce and use the technical notions of investing flow and short intermediate flow; these are some special cases of packet flows that we use in our adversarial constructions that consist of inductive phases. Roughly speaking, an investing flow injects packets in a phase which will remain in the system till the beginning of the next phase, in order to guarantee the induction hypothesis for the next phase; on the other hand, short intermediate flows 
consist of packets injected on judiciously chosen paths of the network and their role is to block all packets of the investing flows (so that the latter will indeed remain in the system).

\subsection{Related Work and Comparison}

Composing Protocols and Objects. The issue of composing distributed protocols (resp., objects) to obtain other protocols (resp., objects), and the properties of the resulting (composed) protocols (resp., objects), has a rich record in Distributed Computing Theory (see, e.g., [10]). For example, Fernández et al. 6] study techniques for the composition of (identical) causal DSM systems from smaller modules each individually satisfying causality. Herlihy and Wing [9] establish that a composition of linearizable memory objects (possibly distinct), each managed by its own protocols, preserves linearizability. In the community of Security Protocols, the statement that security is not compositional is considered to be folklore [11.

Adversarial Queueing Theory. The model of Adversarial Queueing Theory was developed by Borodin et al. [3] as a more realistic model that replaces traditional stochastic assumptions made in Queueing Theory (cf. [4]) by more robust, worst-case ones. Subsequently, the Adversarial Queueing Theory model, and corresponding stability and instability issues, received a lot of interest and attention (see, e.g., $125 \mid 812]$ ).

Stability and Instability Results. The universal stability of SIS, LIS, NTS and FTG was established by Andrews et al. [1. Section 2.1]. The instability of FIFO (on a specific network) was first established by Andrews et al. 11, Theorem 2.10]. Lower bounds of $0.85,0.84$ and 0.8357 on the instability threshold of FIFO (in the model of Adversarial Queueing Theory) were presented before by Andrews et al. [1, Theorem 2.10], Goel [8] and Diaz et al. [5, Theorem 3]. To the best of our knowledge, no previous work addressed the stability and instability properties of networks consisting of queues using multiple contention-resolution protocols.

Summary. For purpose of completeness and comparison, we summarize, in Table 2, all results shown in this work and in [1] that provide bounds on stability and instability properties of the universally stable, greedy contention-resolution protocols, and their compositions, that we considered.

Table 2. Range of injection rates for which the composition of the two protocols is unstable on some network. We denote US the universally stable compositions

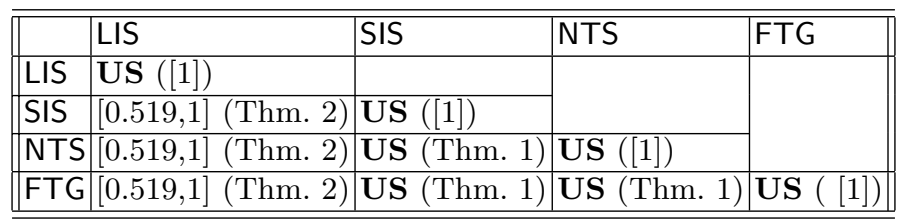




\section{Preliminaries}

The definition of a bounded adversary $\mathcal{A}$ of rate $(r, b)$ (where $b \geq 1$ is a natural number and $0<r<1$ ) in the Adversarial Queueing Theory model 3$]$ requires that for any edge $e$ and any interval $I$, the adversary injects no more than $r|I|+b$ packets during $I$ that require edge $e$ at their time of injection. Such a model allows for adversarial injection of packets that are "bursty" using the integer $b>0$. Say that a packet $p$ requires an edge $e$ at time $t$ if $e$ lies on the path from its position at time $t$ to its destination.

This definition for the adversary is used in Section 3 for proving that specific compositions of protocols are universally stable. On the other hand, when we consider adversarial constructions for proving instability of compositions of specific protocols (Section 4) and FIFO protocol (Section 5) in which we want to derive lower bounds, using an adversary with zero "burstiness" (that is, taking $b=0$ ) results in more simplified proofs. Thus, for these purposes, we say that an adversary $\mathcal{A}$ has injection rate $r$ if for every $t \geq 1$, every interval $I$ of $t$ steps, and every edge $e$, it injects no more than $r|t|$ packets during $I$ that require edge $e$ at the time of their injection. Clearly, an instability result for an adversary with no burstiness $(b=0)$ applies also to an adversary that may use burstiness $(b \geq 0)$. Also, for simplicity, and in a way similar to that in [1], we omit floors and ceilings and sometimes count time steps and packets roughly. This only results to loosing small additive constants while we gain in clarity.

In order to formalize the behavior of a network under the Adversarial Queueing model, we use the notions of system and system configuration. A triple of the form $(G, \mathcal{A}, P)$ where $G$ is a network, $\mathcal{A}$ is the adversary and $P$ is the used protocol on the network queues is called a system. Furthermore, the configuration $C^{t}$ of a system $(G, \mathcal{A}, P)$ in every time step $t$ is a collection of sets $\left\{S_{e}^{t}: e \epsilon G\right\}$, such that $S_{e}^{t}$ is the set of packets waiting in the queue of the edge $e$ at the end of step $t$. If the current system configuration is $C^{t}$, then we can go to the system configuration $C^{t+1}$ for the next time step as follows: (i) Addition of new packets to some of the sets $S_{e}^{t}$, each of which has an assigned path in $G$, and (ii) for each non-empty set $S_{e}^{t}$ deletion of a single packet $p \epsilon S_{e}^{t}$ and its insertion into the set $S_{f}^{t+1}$ where $f$ is the edge following $e$ on its assigned path (if $e$ is the last edge on the path of $p$, then $p$ is not inserted into any set.) The time evolution of the system is a sequence of such configurations $C^{1}, C^{2}, \ldots$, such that for all edges $e$ and all intervals $I$, no more than $r|I|+b$ packets are introduced during $I$ with an assigned path containing $e$. An execution of the adversary's construction on a system $(G, \mathcal{A}, P)$ determines the time evolution of the system configuration.

In the constructions of executions in Sections 4 and 5 we split time into phases. In each phase, we study the evolution of the system configuration by considering corresponding time rounds. For each phase, we inductively prove that the number of packets of a specific subset of queues in the system increases in order to guarantee instability. This inductive argument can be applied repeatedly, thus showing instability. In addition, our constructions use networks that can be split into two symmetric parts. Thus, the inductive argument needs to 
be applied twice to establish increase in the number of packets residing at two different queues.

Also, in order to make our inductions work, we assume that there is a sufficiently large number of packets $s_{0}$ in the initial system configuration. This will imply instability results for networks with an empty initial configuration, as established by Andrews et al. [1, Lemma 2.9].

\section{Universally Stable Compositions of Universally Stable Protocols}

In order to prove the following theorem we make the following assumptions and definitions. Let $0<\epsilon<1$ be a real number. We assume that $r=1-\epsilon$, $m$ is the number of network edges, and $d$ is the length of the longest simple directed path in the network. Let us now define a sequence of numbers by the recurrence $k_{j}=\frac{m k_{j-1}+m b}{\epsilon}$, where $k_{1}=\frac{m b}{\epsilon}$. Our techniques here are motivated by corresponding techniques in Andrews et al. [1].

Theorem 1. If the used queueing disciplines in the system are a) SIS and FTG, then the system $(G, \mathcal{A}, \mathrm{SIS}, \mathrm{FTG})$ is stable, no queue ever contains more than $k_{d}$ packets and no packet spends more than $\frac{1}{\epsilon}\left(d b+\sum_{i=1}^{d} k_{i}\right)$ steps in the system, while if they are b) NTS and FTG or c) SIS and NTS, then there are never more than $k_{d}$ packets in the system and no queue contains more than $\frac{1}{\epsilon}\left(k_{d-1}+b\right)$ packets, where $d$ is the length of the longest simple directed path in $G$ and $k_{i}$ is an appropriately defined sequence of numbers.

Proof. (Sketch) In order to prove this theorem, we first show the following two lemmas:

Lemma 1. Let $p$ be a packet waiting in a queue $e$ at time $t$ and suppose there are currently $k-1$ other packets in the system requiring e that have priority over $p$. Then $p$ will cross e within the next $\frac{k+b}{\epsilon}$ steps if the queueing discipline in queue e is SIS, NTS or FTG.

Lemma 2. When a packet $p$ crossing its path arrives at the $j^{\text {th }}$ edge on its path, there are at most $k_{j}-1$ other packets requiring to traverse this edge with priority over $p$, if the used queueing protocol is a) SIS or NTS, b) SIS or FTG, and c) NTS or FTG.

Lemma 1 claims that if a packet $p$ waits in a queue $e$ at time $t$ and there are currently $k-1$ other packets in the system requiring to traverse edge $e$ that have priority over $p$, then $p$ will cross $e$ within the next $\frac{k+b}{\epsilon}$ steps either the queueing discipline of queue $e$ is SIS or NTS or FTG. In Lemma 2, we specialize Lemma 1 taking into account the distance of the queue $e$, in which packet $p$ arrives at time $t$ crossing its specified path, in relation to the first queue in its path. In this case, we prove that if queue $e$ has distance $j$ from the first queue on $p^{\prime} s$ path then there are at most $k_{j}-1$ other packets in the system requiring to traverse 
the edge $e$ that have priority over $p$ when the system queues have as protocols (a) SIS or FTG, (b)NTS or FTG and (c) SIS or NTS.

Then based on these two lemmas, we prove this theorem using contradiction. Firstly, let us assume that there are $k_{d}+1$ packets at some time all requiring the same edge. Then, the packet with the lowest priority of the $k_{d}+1$ packets contradicts Lemma 2. Combining both lemmas, a packet $p$ takes at most $\frac{k_{j}+b}{\epsilon}$ steps to cross the $j^{t h}$ edge on its path. Therefore, the upper bound for delay is $D=\frac{d b+\sum_{i=1}^{d} k_{i}}{\epsilon}$. No packet spends more than $D$ steps in the system.

\section{Unstable Compositions of Universally Stable Protocols}

In this section, we show that the composition of LIS protocol with any of SIS, NTS and FTG protocols is not universally stable. Before proceeding to the adversary constructions for proving instability we give two basic definitions.

Definition 1. We denote $X_{i}$ the set of packets that are injected by the adversary into the system in the $i_{\text {th }}$ round of a phase. These packet sets are characterized as investing flows because the number of their packets that will remain into the system at the end of the phase in which they have been injected, will be a portion of the packets that will be used as the initial ones in the next phase ensuring the reproduction of the induction hypothesis.

Definition 2. We denote $S_{i, k}$ the $k_{t h}$ set of packets the adversary injects into the system in the $i_{\text {th }}$ round of a phase. These packet sets are characterized as short intermediate flows because their only purpose is to block other packet sets by using suitable paths.

Theorem 2. Let $r \geq 0.519$. There is a network $N_{1}$ and an adversary $\mathcal{A}$ of rate $r$, such that the system $\left(N_{1}, \mathcal{A}\right.$, Queueing Disciplines) is unstable, if the used queueing disciplines are a) LIS and SIS, b) LIS and NTS or c) LIS and FTG.

Proof. (Sketch) Part a) Consider the network $N_{1}$ in Figure 1.

Induction Hypothesis: At the beginning of phase $j$, there are $s_{j}$ packets that are queued in the queues $f_{4}^{\prime}, f_{7}^{\prime}, f_{8}^{\prime}$ (in total) requiring to traverse the edges $e_{0}, f_{1}, f_{2}, f_{4}, f_{7}$.

Induction Step: At the beginning of phase $j+1$ there will be more than $s_{j}$ packets that will be queued in the queues $f_{4}, f_{7}, f_{8}$, requiring to traverse the edges $e_{1}, f_{1}^{\prime}, f_{2}^{\prime}, f_{4}^{\prime}, f_{7}^{\prime}$.

The intuition behind this adversary construction and network topology is basically the preservation of the newly injected investing flows in each round of a phase inside the network until the end of the phase during which they have been injected. The tools we use to achieve this goal are the injections of short intermediate flows $\left(S_{i, k}\right)$ and the introduction of parallel edges $\left(f_{4}, f_{5}, f_{6}\right.$ and $\left.f_{7}, f_{8}, f_{9}\right)$ in the network topology. Although the short intermediate flows do not contribute actually to the number of packets that will be used as the initial flow 


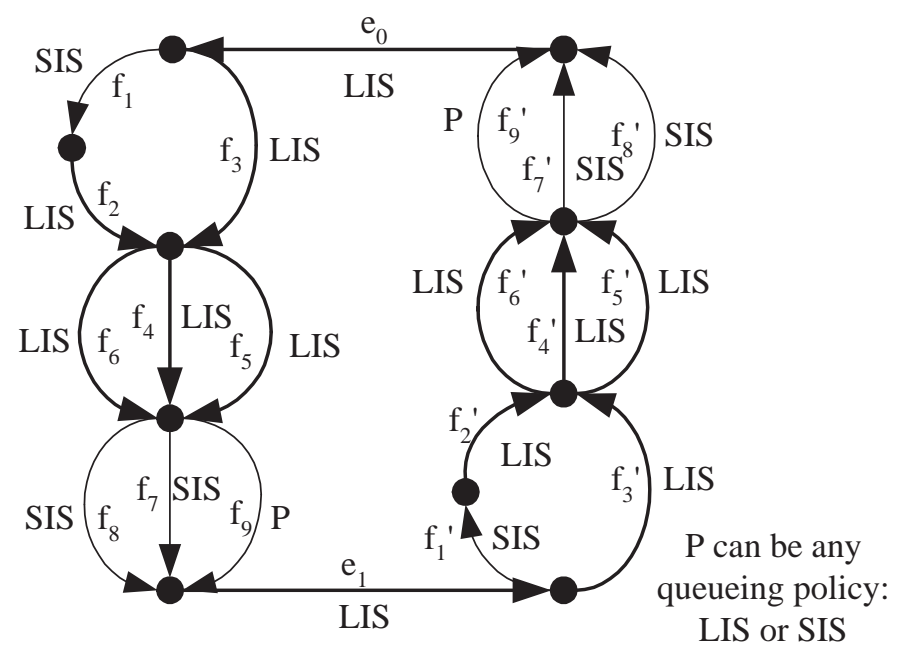

Fig. 1. The network $N_{1}$ running a composition of LIS and SIS

for the next phase, their role is essential as they are used for blocking investing flows of packets in consecutive rounds. Moreover, some of them have another role, too. They block short intermediate flows that have been injected in certain rounds in order they can be used for blocking investing flows in the next round of the one in which they have been injected. Also, an important point we should mention is the use of the short intermediate flow that is injected in the first round of a phase $j\left(S_{1,1}-\right.$ flow $)$ to block a portion of the initial packets that are in the system at the beginning of the phase $\left(s_{j}\right)$ in order these packets to be used twice for blocking investing flow $X_{1}$ to the first and the next round of the current phase. As far as concerns the parallel edges, the purpose of their presence in the network topology is to be guaranteed that the paths of the packet flows injected in the same round do not overlap. The first set of parallel edges $f_{4}, f_{5}, f_{6}$ is used in order short intermediate flows that are injected at the same round to not overlap, while the second set of parallel edges $f_{7}, f_{8}, f_{9}$ is used mainly in order the paths of investing flows and the paths of short intermediate flows that are injected at the same round to not overlap.

We will construct an adversary $\mathcal{A}$ such that the induction step will hold. Proving that the induction step holds, we ensure that the induction hypothesis will hold at the beginning of phase $j+1$ for the symmetric edges with an increased value of $s_{j}, s_{j+1}>s_{j}$. In order to prove that the induction step works, we should consider that there is a large enough number of packets $s_{j}$ in the initial system configuration. During phase $j$ the adversary plays four rounds of injections. The sequence of injections is as follows:

Round 1: It lasts $s_{j}$ time steps. At the beginning of this round, there are $s_{j}$ packets $\left(S-\right.$ flow) in the queues $f_{4}^{\prime}, f_{7}^{\prime}, f_{8}^{\prime}$ (in total) requiring to traverse the edges $e_{0}, f_{1}, f_{2}, f_{4}, f_{7}$. 
Adversary's behavior. During this round the adversary injects in queue $e_{0}$ a set $X_{1}$ of $\left|X_{1}\right|=r s_{j}$ packets wanting to traverse the edges $e_{0}, f_{3}, f_{4}, f_{7}, e_{1}, f_{1}^{\prime}$, $f_{2}^{\prime}, f_{4}^{\prime}, f_{7}^{\prime}$. At the same time, the adversary injects a set $S_{1,1}$ of $\left|S_{1,1}\right|=r s_{j}$ packets in queue $f_{1}$ that require to traverse only the edge $f_{1}$.

At the end of this round, there are $r s_{j}$ packets of $S$-flow in queue $f_{1}$ wanting to traverse the edges $f_{1}, f_{2}, f_{4}, f_{7}$. Also, there is a set $X_{1}$ of $\left|X_{1}\right|=r s_{j}$ packets in queue $e_{0}$ wanting to traverse the edges $e_{0}, f_{3}, f_{4}, f_{7}, e_{1}, f_{1}^{\prime}, f_{2}^{\prime}, f_{4}^{\prime}, f_{7}^{\prime}$.

Round 2: It lasts $r s_{j}$ time steps. Adversary's behavior: During this round the adversary injects a set $X_{2}$ of $\left|X_{2}\right|=r^{2} s_{j}$ packets in queue $e_{0}$ requiring to traverse the edges $e_{0}, f_{3}, f_{4}, f_{8}, e_{1}, f_{1}^{\prime}, f_{2}^{\prime}, f_{4}^{\prime}, f_{7}^{\prime}$. In addition, the adversary injects a set $S_{2,1}$ of $\left|S_{2,1}\right|=r^{2} s_{j}$ packets in queue $f_{2}$ wanting to traverse the edges $f_{2}, f_{5}, f_{7}$.

At the end of this round, there are $r s_{j}$ packets of $X_{1}-$ flow in queue $f_{4}$ wanting to traverse the edges $f_{4}, f_{7}, e_{1}, f_{1}^{\prime}, f_{2}^{\prime}, f_{4}^{\prime}, f_{7}^{\prime}$. Also, there are $r^{2} s_{j}$ packets of $X_{2}-$ flow in queue $e_{0}$ requiring to traverse the edges $e_{0}, f_{3}, f_{4}, f_{8}, e_{1}, f_{1}^{\prime}, f_{2}^{\prime}, f_{4}^{\prime}, f_{7}^{\prime}$ and $r^{2} s_{j}$ packets of $S_{2,1}-$ flow in queue $f_{2}$ wanting to traverse the edges $f_{2}, f_{5}, f_{7}$. Round 3: It lasts $r^{2} s_{j}$ time steps. Adversary's behavior: During this round the adversary injects a set $X_{3}$ of $\left|X_{3}\right|=r^{3} s_{j}$ packets in queue $f_{4}$ wanting to traverse the edges $f_{4}, f_{9}, e_{1}, f_{1}^{\prime}, f_{2}^{\prime}, f_{4}^{\prime}, f_{7}^{\prime}$. Also, the adversary injects a set $S_{3,1}$ of $\left|S_{3,1}\right|=r^{3} s_{j}$ packets in queue $f_{5}$ wanting to traverse the edges $f_{5}, f_{7}$. Finally, the adversary injects a set $S_{3,2}$ of $\left|S_{3,2}\right|=r^{3} s_{j}$ packets in queue $f_{2}$ wanting to traverse the edges $f_{2}, f_{6}, f_{8}$.

At the end of this round, there are $r s_{j}$ packets of $X_{1}-$ flow in queues $f_{4}, f_{7}$ in total $\left(r s_{j}-r^{2} s_{j}\right.$ packets in queue $f_{4}$ and $r s_{j}$ packets in queue $\left.f_{7}\right)$ wanting to traverse the edges $f_{4}, f_{7}, e_{1}, f_{1}^{\prime}, f_{2}^{\prime}, f_{4}^{\prime}, f_{7}^{\prime}$. Also, in queue $f_{4}$ there are $r^{2} s_{j}$ packets of $X_{2}-$ flow requiring to traverse the edges $f_{4}, f_{8}, e_{1}, f_{1}^{\prime}, f_{2}^{\prime}, f_{4}^{\prime}, f_{7}^{\prime}$ and $r^{3} s_{j}$ packets of $X_{3}-$ flow wanting to traverse the edges $f_{4}, f_{9}, e_{1}, f_{1}^{\prime}, f_{2}^{\prime}, f_{4}^{\prime}, f_{7}^{\prime}$. Moreover, there are $r^{3} s_{j}$ packets of $S_{3,1}-$ flow in queue $f_{5}$ requiring to traverse the edges $f_{5}, f_{7}$ and $r^{3} s_{j}$ packets of $S_{3,2}-$ flow in queue $f_{2}$ requiring to traverse the edges $f_{2}, f_{6}, f_{8}$.

Round 4: It lasts $r^{3} s_{j}$ time steps. Adversary's behavior: During this round, the adversary injects a set $X_{4}$ of $\left|X_{4}\right|=r^{4} s_{j}$ packets in queue $f_{3}$ wanting to traverse the edges $f_{3}, f_{4}, f_{7}, e_{1}, f_{1}^{\prime}, f_{2}^{\prime}, f_{4}^{\prime}, f_{7}^{\prime}$. Besides the short intermediate flows $\left(S_{3,1}, S_{3,2}\right)$ that have been injected into the system during the previous round and still remain into the system at the beginning of this round, the system configuration at the beginning of round 4 also consists of the investing flows $X_{1}, X_{2}, X_{3}$ that have been injected into the system during the previous rounds. During this round, these investing flows along with the investing flow that is injected into the system during this round $\left(X_{4}-\right.$ flow) continue to remain into the system independently of the adversarial injection rate $r$ because they are blocked by the short intermediate flows $S_{3,1}, S_{3,2}$.

At the end of this round, the number of packets in queues $f_{4}, f_{7}, f_{8}$ requiring to traverse the edges $e_{1}, f_{1}^{\prime}, f_{2}^{\prime}, f_{4}^{\prime}, f_{7}^{\prime}$ is

$$
s_{j+1}=\left|X_{1}\right|+\left|X_{2}\right|+\left|X_{3}\right|+\left|X_{4}\right|=r s_{j}+r^{2} s_{j}+r^{3} s_{j}+r^{4} s_{j}
$$


In order to have instability, we must have $s_{j+1}>s_{j}$. Therefore from (1), we should have $r s_{j}+r^{2} s_{j}+r^{3} s_{j}+r^{4} s_{j}>s_{j}$, i.e. $r \geq 0.519$. This argument can be repeated for an infinite number of phases ensuring that the number of packets at the end of a phase will be bigger than at the beginning of the phase.

Part b) This part of the theorem can be proved similarly to the previous part. One difference here is the replacement of the protocol of queues that use SIS by NTS. The topology of the used network $N_{2}$ and the adversary construction for proving instability of the system $\left(N_{2}, \mathcal{A}\right.$, LIS, NTS) are similar to the first part of the theorem. Especially, short intermediate flows have the same blocking effects as before over investing flows because their injection in the same queues as before are enough to guarantee their priority over investing flows when they conflict in queues that use NTS.

Part c) This part of the theorem can be proved similarly to the previous parts. Thus, the construction of the adversary $\mathcal{A}$ for proving instability of the system $\left(N_{3}, \mathcal{A}\right.$, LIS,FTG $)$ is similar to the other parts. As far as concerns the network topology a difference here is that the queues, that have as protocol SIS in the first part, are now using FTG. Another difference that concerns the used network topology is that it contains additional paths that start at FTG queues and have sufficient lengths, such that the injected short intermediate packet flows have the same blocking effects over the injected investing packet flows, as in the proofs of the previous parts, when they conflict in queues that use FTG.

\section{A Lower Bound for FIFO Instability}

In this section, we present an adversary construction that lowers significantly the injection rate bound for which FIFO is unstable to 0.749 on the network that we consider in Figure2.

Theorem 3. Let $r \geq 0.749$. There is a network $\mathcal{N}$ and an adversary $\mathcal{A}$ of rate $r$, such that the $(\mathcal{N}, \mathcal{A}, \mathrm{FIFO})$ system is unstable.

Proof. (Sketch) We consider the network $\mathcal{N}$ in Figure 2.

Induction Hypothesis: At the beginning of phase $j$, there are $s_{j}$ packets that are queued in the queues $e_{0}, f_{3}^{\prime}, f_{4}^{\prime}, f_{5}^{\prime}, f_{6}^{\prime}$ (in total) requiring to traverse the edges $e_{0}, f_{1}, f_{3}, f_{5}$, all these packets are able to depart from their initial edges to the symmetric part of the network $\left(f_{1}, f_{3}, f_{5}\right)$ as a continuous flow in $s_{j}$ time steps, and the number of packets that are queued in queues $f_{4}^{\prime}, f_{6}^{\prime}$ is bigger than the number of packets that are queued in queues $f_{3}^{\prime}, f_{5}^{\prime}$.

Induction Step: At the beginning of phase $j+1$ there will be more than $s_{j}$ packets $\left(s_{j+1}\right.$ packets) that will be queued in the queues $f_{3}, f_{5}, f_{4}, f_{6}, e_{1}$ (in total) requiring to traverse the edges $e_{1}, f_{1}^{\prime}, f_{3}^{\prime}, f_{5}^{\prime}$, all of which will be able to depart from their initial edges to the symmetric part of the network $\left(f_{1}^{\prime}, f_{3}^{\prime}, f_{5}^{\prime}\right)$ in $s_{j+1}$ time steps as a continuous flow and the number of packets that will be queued in queues $f_{4}, f_{6}$ will be bigger than the number of packets that will be queued in queues $f_{3}, f_{5}$. 


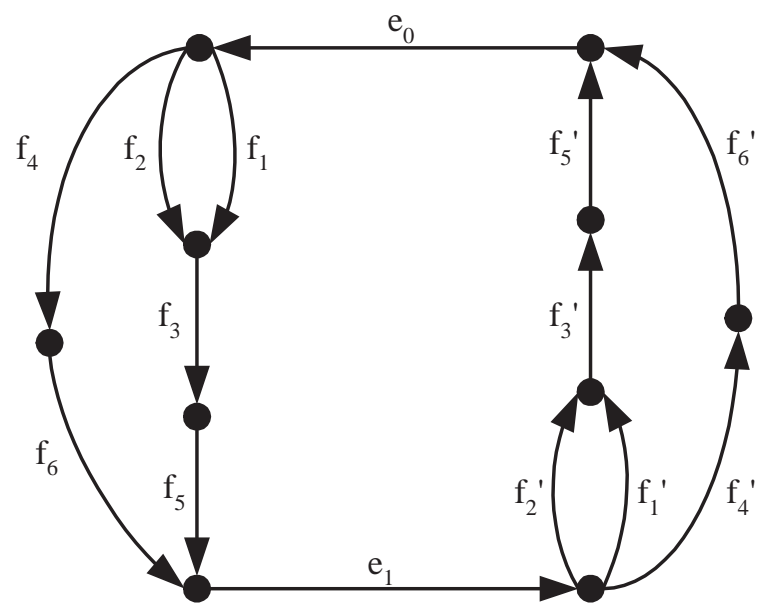

Fig. 2. FIFO network $\mathcal{N}$

Notice that our inductive argument claims that if at the beginning of phase $j$ all $s_{j}$ packets, that are queued in queues $e_{0}, f_{3}^{\prime}, f_{4}^{\prime}, f_{5}^{\prime}, f_{6}^{\prime}$ requiring to traverse the edges $e_{0}, f_{1}, f_{3}, f_{5}$, manage to traverse their initial edges in $s_{j}$ time steps as a continuous flow, then at the beginning of phase $j+1$ all $s_{j+1}$ packets, that will be queued in queues $f_{3}, f_{5}, f_{4}, f_{6}, e_{1}$ requiring to traverse the edges $e_{1}, f_{1}^{\prime}, f_{3}^{\prime}, f_{5}^{\prime}$, will be able to traverse their initial edges in $s_{j+1}$ time steps as a continuous flow. This argument guarantees the reproduction of the induction hypothesis in queues $f_{3}, f_{5}, f_{4}, f_{6}, e_{1}$ even if there are flows (in particular in queues $f_{3}, f_{4}, f_{5}$ ) that do not want to traverse the edges $e_{1}, f_{1}^{\prime}, f_{3}^{\prime}, f_{5}^{\prime}$, the packets of which are regularly spread among the packets that want to traverse these edges. Furthermore, this argument implies the third part of the inductive argument, which claims that if at the beginning of phase $j$, the number of packets that are queued in queues $f_{4}^{\prime}, f_{6}^{\prime}$ is bigger than the number of packets that are queued in queues $f_{3}^{\prime}, f_{5}^{\prime}$, then at the beginning of phase $j+1$ the number of packets that will be queued in queues $f_{4}, f_{6}$ will be bigger than the number of packets that will be queued in queues $f_{3}, f_{5}$. This happens because in the first round of the adversary's construction we inject packets in queue $f_{4}^{\prime}$ and if the third part of the induction hypothesis does not hold, then we cannot guarantee that all the initial $s_{j}$ packets will depart their initial edges to the edges $f_{1}, f_{3}, f_{5}$ in $s_{j}$ time steps as a continuous flow. However, we include it into the induction hypothesis for readability reasons.

We will construct an adversary $\mathcal{A}$ such that the induction step will hold. Proving that the induction step holds, we ensure that the induction hypothesis will hold at the beginning of phase $j+1$ for the symmetric edges with an increased value of $s_{j}$ packets, $s_{j+1}>s_{j}$. From the induction hypothesis, initially, there are $s_{j}$ packets (called $S-$ flow) in the queues $e_{0}, f_{3}^{\prime}, f_{4}^{\prime}, f_{5}^{\prime}, f_{6}^{\prime}$ requiring to traverse the edges $e_{0}, f_{1}, f_{3}, f_{5}$. In order to prove the induction step, it is assumed that there is a set $S$ with a large enough number of $|S|=s_{j}$ packets in the 
initial system configuration. During phase $j$ the adversary plays three rounds of injections. The sequence of injections is as follows:

Round 1: It lasts $s_{j}$ time steps. Adversary's behavior: During these steps, the adversary injects a set $X$ of $|X|=r s_{j}$ packets in queue $f_{4}^{\prime}$ wanting to traverse the edges $f_{4}^{\prime}, f_{6}^{\prime}, e_{0}, f_{2}, f_{3}, f_{5}, e_{1}, f_{1}^{\prime}, f_{3}^{\prime}, f_{5}^{\prime}$ and a set $S_{1}$ of $\left|S_{1}\right|=r s_{j}$ packets in $f_{1}$ wanting to traverse $f_{1}$.

At the end of this round, all the packets of the set $X$ are queued in $e_{0}$, while in queue $f_{1}$ remains a set $S_{r e m}$ of $\left|S_{r e m}\right|=\frac{r s_{j}}{r+1}$ packets from the set $S$ and a set $S_{1, \text { rem }}$ of $\left|S_{1, \text { rem }}\right|=\frac{r^{2} s_{j}}{r+1}$ packets from the set $S_{1}$ mixed on a proportion equal to their initial proportion of their sizes (fair mixing property).

Round 2: It lasts $r s_{j}$ steps. Adversary's behavior: The adversary injects a set $Y$ of $|Y|=r^{2} s_{j}$ packets in queue $f_{4}^{\prime}$ requiring to traverse the edges $f_{4}^{\prime}, f_{6}^{\prime}, e_{0}, f_{4}$, $f_{6}, e_{1}, f_{1}^{\prime}, f_{3}^{\prime}, f_{5}^{\prime}$. At the same time, the adversary injects a set $S_{2}$ of $\left|S_{2}\right|=r^{2} s_{j}$ packets in $f_{2}$ wanting to traverse $f_{2}$, a set $S_{3}$ of $\left|S_{3}\right|=r^{2} s_{j}$ packets in $f_{3}$ wanting to traverse $f_{3}$, and a set $S_{4}$ of $\left|S_{4}\right|=r^{2} s_{j}$ packets in $f_{5}$ wanting to traverse $f_{5}$.

At the end of this round all the packets of the set $Y$ are queued in queue $e_{0}$. Also, a set $X_{r e m, f_{2}}$ of $\left|X_{r e m, f_{2}}\right|=\frac{r^{2} s_{j}}{r+1}$ packets from the set $X$ and a set $S_{2, \text { rem, } f_{2}}$ of $\left|S_{2, r e m, f_{2}}\right|=\frac{r^{3} s_{j}}{r+1}$ packets from the set $S_{2}$ remain in queue $f_{2}$ mixed on a proportion equal to their initial proportion of their sizes. Furthermore a set $X_{r e m, f_{3}}$ of $\left|X_{r e m, f_{3}}\right|=\frac{r^{3} s_{j}+r s_{j}}{(r+1)\left(r^{2}+r+2\right)}$ packets from the set $X$, a set $S_{r e m, f_{3}}$ of $\left|S_{r e m, f_{3}}\right|=\frac{r^{3} s_{j}+r s_{j}}{(r+1)\left(r^{2}+r+2\right)}$ packets from the set $S$ and a set $S_{3, r e m}$ of $\left|S_{3, \text { rem }}\right|=\frac{r^{4} s_{j}+r^{2} s_{j}}{r^{2}+r+2}$ packets from the set $S_{3}$ remain in queue $f_{3}$ mixed on the proportion of the sizes with which they arrive in queue $f_{3}$ during this round. Finally, $\frac{r^{4} s_{j}+r^{2} s_{j}}{\left(r^{2}+r+2\right)\left(r^{3}+r^{2}+2 r+2\right)}$ packets from the set $X, \frac{r^{4} s_{j}+r^{2} s_{j}}{\left(r^{2}+r+2\right)\left(r^{3}+r^{2}+2 r+2\right)}$ packets from the set $S$, and $\frac{r^{5} s_{j}+r^{3} s_{j}}{r^{3}+r^{2}+2 r+2}$ packets from the set $S_{4}$ remain in queue $f_{5}$ mixed on the proportion of the sizes with which they arrive in queue $f_{3}$ during this round.

Round 3: It lasts $r^{2} s_{j}$ time steps. Adversary's behavior: During this round the adversary injects a set $S_{5}$ of $\left|S_{5}\right|=r^{3} s_{j}$ packets in queue $f_{4}$ requiring to traverse the edge $f_{4}$ and a set $Z$ of $|Z|=r^{3} s_{j}$ packets in queue $f_{6}$ requiring to traverse the edges $f_{6}, e_{1}, f_{1}^{\prime}, f_{3}^{\prime}, f_{5}^{\prime}$.

At the end of this round a set $Y_{\text {rem }}$ of $\left|Y_{\text {rem }}\right|=\frac{r^{3} s_{j}}{r+1}$ packets from the set $Y$ and a set $S_{5, \text { rem }}$ of $\left|S_{5, \text { rem }}\right|=\frac{r^{4} s_{j}}{r+1}$ packets from the set $S_{5}$ remain in queue $f_{4}$ mixed on a proportion equal to their initial proportion of their sizes. Furthermore, a set $Y_{r e m, f_{6}}$ of $\left|Y_{r e m, f_{6}}\right|=\frac{r^{4} s_{j}}{(r+1)\left(r^{2}+r+1\right)}$ packets from the set $Y$ and a set $Z_{r e m, f_{6}}$ of $\left|Z_{r e m, f_{6}}\right|=\frac{r^{5} s_{j}}{r^{2}+r+1}$ packets from the set $Z$ are queued in queue $f_{6}$ mix on the proportion of the sizes with which they arrive in queue $f_{6}$ during this round.

The total number of packets in queue $f_{3}$ at the beginning of round 3 is

$$
\left|T_{1}\right|=\left|X_{r e m, f_{3}}\right|+\left|S_{r e m, f_{3}}\right|+\left|S_{3, r e m}\right|=\frac{\left(r^{5}+r^{4}+3 r^{3}+r^{2}+2 r\right) s_{j}}{(r+1)\left(r^{2}+r+2\right)}
$$


However, $\left|T_{1}\right| \geq r^{2} s_{j}, \forall r$. Thus, a number of $X_{r e m, f_{3}}, S_{r e m, f_{3}}, S_{3, \text { rem }}$ packets will remain in queue $f_{3}$ at the end of round 3. This number is $\left|T_{2}\right|=\frac{\left(2 r-r^{2}-r^{4}\right) s_{j}}{(r+1)\left(r^{2}+r+2\right)}$. From this number $\left|S_{3, r e m, f_{3}}\right|=\frac{\left(2 r^{2}-r^{3}-r^{5}\right) s_{j}}{\left(r^{2}+r+2\right)^{2}}$ packets belong to the set $S_{3}$. In addition, the total number of packets that are in $f_{2}$ at the end of round 2 is $\left|T_{3}\right|=r^{2} s_{j}$ that is equal to the round's time duration. Thus, all the packets that belong to the set $X$ and were in $f_{2}$ at the end of round $2\left(\left|X_{\text {rem, } f_{2}}\right|\right)$ traverse now $f_{2}$ and arrive to $f_{3}$ where they are blocked.

In order to have instability the number of packets that are queued in $f_{3}, f_{4}, f_{5}$, $f_{6}, e_{1}$ requiring to traverse the edges $e_{1}, f_{1}^{\prime}, f_{3}^{\prime}, f_{5}^{\prime}$ at the end of this round, $s_{j+1}$, should be more than the initial $s_{j}$ packets that were queued in the system in corresponding queues at the beginning of round 1. Therefore, it should hold

$$
|Z|+|Y|+\left|X_{\text {rem, } f_{2}}\right|+\left|X_{\text {rem }, f_{3}}\right|+\left|X_{\text {pass }, f_{3}}\right|-\left|T_{\text {round }_{3}}\right|>s_{j}
$$

Substituting in (3), we take

$$
r^{3} s_{j}+\frac{r^{2} s_{j}}{r+1}+\frac{r^{3} s_{j}+r s_{j}}{(r+1)\left(r^{2}+r+2\right)}+\frac{r^{4} s_{j}+r^{2} s_{j}}{\left(r^{2}+r+2\right)\left(r^{3}+r^{2}+2 r+2\right)}>s_{j}
$$

This holds for $r \geq 0.749$. Now in order to conclude the proof we prove:

Lemma 3. For $r \geq 0.686$, the number of packets that remain in queues $f_{3}, f_{5}$ $\left(Q\left(f_{3}\right), Q\left(f_{5}\right)\right)$ at the end of round 3 is less than or equal to the number of packets that remain in queues $f_{4}, f_{6}$ at the end of round $3\left(Q\left(f_{4}\right), Q\left(f_{6}\right)\right)$.

Notice that we have, till now, managed to reproduce the induction hypothesis in queues $f_{3}, f_{5}, f_{4}, f_{6}, e_{1}$ but with some packet flows (in particular in queues $f_{4}, f_{3}, f_{5}$ ) having empty spaces (packets that don't want to traverse the edges $\left.e_{1}, f_{1}^{\prime}, f_{3}^{\prime}, f_{5}^{\prime}\right)$. In order for the induction step to work we must show that all the packets in these queues will manage to depart to the symmetric part of the network $\left(f_{1}^{\prime}, f_{3}^{\prime}, f_{5}^{\prime}\right)$ in $s_{j+1}$ time steps as a continuous flow. As we have shown above all the packets that are queued in queue $e_{1}$ want to traverse the edges $e_{1}, f_{1}^{\prime}, f_{3}^{\prime}, f_{5}^{\prime}$ and their flow is continuous without empty spaces (packets that do not want to traverse the edges $e_{1}, f_{1}^{\prime}, f_{3}^{\prime}, f_{5}^{\prime}$ ). Also, from Lemma 3 the number of packets in queues $f_{4}, f_{6}$ is bigger than the number of packets in queues $f_{3}, f_{5}$. Furthermore, the packets that are queued in queue $f_{6}$ can be seen as a continuous flow that wants to traverse the edges $e_{1}, f_{1}^{\prime}, f_{3}^{\prime}, f_{5}^{\prime}$, while the set of packets in queue $f_{4}$ consists of packets that want to traverse the edges $e_{1}, f_{1}^{\prime}, f_{3}^{\prime}, f_{5}^{\prime}\left(Y_{\text {rem }}\right)$ and packets that are injections which require to traverse a single edge $\left(S_{5, \mathrm{rem}}\right)$, which can be considered as empty spaces. Because of that we should show that all the $Y_{\text {rem }}$ packets manage to leave the edge $f_{4}$ during the $s_{j+1}$ time steps. We formally establish:

Lemma 4. For any injection rate $r$, all the $Y_{\text {rem }}$ packets manage to leave the edge $f_{4}$ during $s_{j+1}$ time steps.

We have so far established two sufficient constraints on $r$ for instability, namely that $r \geq 0.749$ and $r \geq 0.686$. Clearly, taking $r \geq \max \{0.749,0.686\}=0.749$ suffices for instability of the network $\mathcal{N}$ in the constructed execution. This concludes our proof. 


\section{Discussion and Directions for Further Research}

Our work opens up the study of the stability and instability properties of heterogeneous communication networks with multiple contention-resolution protocols running on top of them. A fundamental question that arises in this setting is whether there exists a structural explanation of the differences we have observed regarding the stability of different compositions of universally stable protocols. Is there any deep reason for the composition of LIS with another contentionresolution protocol to be unstable? Also, for the unstable compositions of pairs of protocols, can we characterize the graphs on which the composition is unstable? A corresponding characterization for networks on which a single greedy protocol is running (as opposed to a composition of greedy protocols) in terms of graph minors has been developed in [1] Section 3.2].

\section{References}

1. M. Andrews, B. Awerbuch, A. Fernandez, J. Kleinberg, T. Leighton, and Z. Liu, "Universal Stability Results for Greedy Contention-Resolution Protocols," Journal of the ACM, Vol. 48, No. 1, pp. 39-69, January 2001.

2. M. Andrews, A. Férnandez, A. Goel and L. Zhang, "Source Routing and Scheduling in Packet Networks," Proceedings of the 42nd Annual IEEE Symposium on Foundations of Computer Science, pp. 168-177, October 2002.

3. A. Borodin, J. Kleinberg, P. Raghavan, M. Sudan and D. Williamson, "Adversarial Queueing Theory," Journal of the ACM, Vol. 48, No. 1, pp. 13-38, January 2001.

4. H. Chen and D. D. Yao, Fundamentals of Queueing Networks, Springer, 2000.

5. J. Diaz, D. Koukopoulos, S. Nikoletseas, M. Serna, P. Spirakis and D. Thilikos, "Stability and Non-Stability of the FIFO Protocol," Proceedings of the 13th Annual ACM Symposium on Parallel Algorithms and Architectures, pp. 48-52, July 2001.

6. A. Férnandez, E. Jiménez and V. Cholvi, "On the Interconnection of Causal Memory Systems," Proceedings of the 19th Annual ACM Symposium on Principles of Distributed Computing, pp. 163-170, July 2000.

7. S. Floyd and V. Paxson, "Difficulties in Simulating the Internet," IEEE/ACM Transactions on Networking, Vol. 9, No. 4, pp. 392-403, August 2001.

8. A. Goel, "Stability of Networks and Protocols in the Adversarial Queueing Model for Packet Routing," Networks, Vol. 37, No. 4, pp. 219-224, 2001.

9. M. P. Herlihy and J. Wing, "Linearizability: A Correctness Condition for Concurrent Objects," ACM Transactions on Programming Languages and Systems, Vol. 12, No. 3, pp. 463-492, 1990.

10. N. Lynch, Distributed Algorithms, Morgan Kaufmann, 1996.

11. J. Mitchell, Email Communication to I. Lee, April 2002.

12. P. Tsaparas, Stability in Adversarial Queueing Theory, M.Sc. Thesis. Department of Computer Science, University of Toronto, 1997. 